\section{$\alpha$-L-Arabinofuranosidase Activity during Development and Ripening of Normal and ACC Synthase Antisense Tomato Fruit}

\author{
Gabriel O. Sozzi and Adela A. Fraschina \\ Departamento de Biología Aplicada y Alimentos, Facultad de Agronomía, \\ Universidad de Buenos Aires, Avda, San Martín 4453, C1417DSE, Buenos \\ Aires, Argentina
}

\begin{abstract}
Agustín A. Navarro and Osvaldo Cascone
Departamento de Microbiología, Biotecnología e Inmunología, Facultad de Farmacia y Bioquímica, Universidad de Buenos Aires, Junín 956 C1113AAD, Buenos Aires, Argentina
\end{abstract}

L. Carl Greve and John M. Labavitch ${ }^{1}$

Department of Pomology, University of California-Davis, Wickson Hall, One Shields Avenue, Davis, CA 95616-8683

\section{Additional index words. Lycopersicon esculentum, ethylene, glycosidase}

\begin{abstract}
L-Arabinofuranosidases ( $\alpha$-Af) are plant enzymes that have the capacity to release terminal arabinofuranosyl residues from a wide variety of pectic and hemicellulosic polymers, as well as different glycoconjugates. Our interest in $\alpha$-Af is related to its potential role in ripening-related loss of arabinose from tomato fruit cell walls. Using both control (cv. VF 36) and ACC synthase antisense (A11.1) tomatoes (Lycopersicon esculentum Mill.), we demonstrate that tomato $\alpha$-Af activity is present during the entire ontogeny of the fruit. Immature 10-day-old fruit displayed 6-fold more $\alpha$-Af activity on a per gram fresh weight basis, than mature green fruit. In VF 36 fruit, $\alpha$-Af activity increased $45 \%$ from mature green 4 ( 48 days post anthesis) to light red stages ( 55 days) when fruit ripened on the vine. In contrast, no similar increase was detected in ACC synthase antisense fruit that do not ripen in the same time frame. However, when A11.1 fruit were detached at 48 days after anthesis and treated continuously with $100 \mathrm{~mL} \cdot \mathrm{L}^{-1}$ ethylene the fruit ripened and $\alpha$-Af increased, as in ripening normal fruit. The $\alpha$-Af activity pattern is similar to that reported for tomato $\beta$-galactosidases. The increasing $\alpha$-Af activity during ripening and the decreased activity in antisense ACC synthase fruit after reaching the mature green stage suggest a role for ethylene in the ripening-related synthesis or activation of this enzyme.
\end{abstract}

Texture is a major attribute that has a strong effect on consumer perception of tomato fruit quality. Different factors affect tomato textural properties, among them cell wall polysaccharide composition (Barrett et al., 1998). Most of the covalent modifications in cell wall polysaccharides result from the activity of a set of hydrolases that may participate in a concerted enzymatic action (Fischer and Bennett, 1991). A substantial decrease in cell wall-bound galactosyl and arabinosyl residues is one of the most evident cell wall compositional changes during fruit ripening (Gross and Sams, 1984; Seymour et al., 1990). These neutral sugar components usually occur as side chains (5-arabinan, 4-

Received for publication 16 Apr. 2001. Accepted for publication: 10 Sept. 2001. This study was partially funded by grants from the Universidad de Buenos Aires (UBACyT, TG043), the Agencia Nacional de Promoción Científica y Tecnológica (Project 08-04650), and Fundación Antorchas (Project 13887-22).

${ }^{1}$ To whom reprint requests should be addressed. Fax: (530) 752 8502. E-mail address: jmlabavitch@ ucdavis.edu galactan, arabinogalactan) attached to rhamnosyl residues of the rhamnogalacturonan backbone (Carpita and Gibeaut, 1993). Terminal $\alpha$-L-arabinosyl units are also present in the arabinoxyloglucans of the Solanaceae (York et al., 1996) and in the substantial carbohydrate component of arabinogalactan proteins (AGPs; Cassab, 1998 and references cited therein).

$\beta$-galactosidases in growing and ripening tomatoes have been studied in relationship to their potential for removing galactosyl residues from cell wall polymers (Carey et al., 1995; Carrington and Pressey, 1996; Pressey, 1983; Sozzi et al., 1998a) and their impact in tomato fruit metabolism and softening is now being investigated using transgenic plants (Smith and Gross, 2000; Smith et al., 1998). However, the loss of cell wall arabinosyl residues has received much less attention. Cell wall arabinose loss continues after harvest in mature rin tomato fruit even though they do not soften (Gross and Wallner, 1979). However, there have been no studies of the effect of the specific suppression of ethylene synthesis on cell wall arabinose change to date.
The cell wall is by far the major arabinosecontaining structure of plants. Arabinose is the primary neutral sugar residue lost, during maturation, in some commercially important fruits, such as pears (Pyrus communis L.), peaches and nectarines [Prunus persica (L.) Batsch], blueberries (Vaccinium corymbosum L.) and strawberries (Fragaria $\times$ ananassa Duch.) (Gross and Sams, 1984) and significant changes in arabinose content have also been detected in avocado (Persea americana Mill.) (Redgwell et al., 1997). In tomato, the most intensively studied fruit over the last decades, $25 \%$ of the cell wall arabinose may be released in the 4 - to 5 -d period between the turning and the red ripe stages (Gross and Sams, 1984). Several polysaccharide types might be involved. Limited breakdown of xyloglucan occurs early in tomato fruit ripening and pectins are hydrolyzed increasingly as fruit become red ripe (Brummell et al., 2000). The only report of AGP metabolism during tomato fruit development describes accelerated synthesis of AGP carbohydrate early in ripening (Huysamer et al., 1997).

Fruit softening is not the only reason to study the enzymes potentially capable of removing arabinosyl residues. The arabinogalactan proteins may be involved in important processes during growth and development, including modifications of cell wall composition and cell-to-cell associations (Cassab, 1998). Moreover, Priem et al. (1993) reported a tomato protein-associated $N$-glycan containing an arabinosyl residue and suggested that $N$-glycans could have a key role in the regulation of tomato fruit senescence. Thus, the mechanisms responsible for the release of arabinosyl residues could also modulate ripening-related biological processes in addition to softening.

$\alpha$-L-Arabinofuranosidase [ $\alpha$-L-arabinofuranoside arabinofuranohydrolase, EC 3.2.1.55; $\alpha$-Af] has been widely studied in microorganisms (e.g., Saha, 2000) and plant tissues (e.g., Konno et al., 1987). It has also been detected in several fruits [e.g., Japanese pear (Pyrus serotina Rehder) Tateishi et al., 1996; goldenberry (Physalis peruviana L.); Trinchero et al., 1999], and in both the pericarp (Campbell et al., 1990; Sozzi et al., 1998b) and locule (Cheng and Huber, 1997) cell walls of tomato. This paper describes work examining the presence of $\alpha$-Af during tomato growth and ripening and uses ACC synthase antisense fruit to determine whether ethylene influences $\alpha$-Af activity during ripening.

\section{Materials and Methods}

Plant material and chemicals. Control tomato seeds (cv. VF36) and transgenic seeds (called A11.1, which are in the 'VF36' genetic background) expressing antisense ACC-synthase RNA were obtained from Athanasios Theologis [Plant Gene Expression Center, Univ. of California-Berkeley, U.S. Dept. of Agriculture (USDA), Albany, Calif.]. Forty plants of each type were grown under daylight in 15-L plastic pots in a greenhouse at the Univ. of California, Davis. Tomato plants 
were trellised and fertilized as described by Cadahía López (1995). Flowers of control and antisense ACC synthase plants were tagged at anthesis. Both control and antisense fruit were harvested at different phenology stages. Only the early-set proximal fruit of each truss were selected to minimize alterations due to different hormonal fluxes and variations of assimilate import. Forty-eight-day-old (mature green stage) control and antisense fruit were harvested and stored at $20 \pm 1{ }^{\circ} \mathrm{C}$ in humidified air and diffuse light until used. Ripeness stages in normal 'VF36' fruit were established in comparison with the tomato color chart of the USDA (1976), as follows: mature green 4 (day 48); breaker (day 50); light red (day 55); red ripe (day 57); over ripe (day 70 ). Untreated antisense fruit do not ripen and so comparisons with ripening VF36 fruit are based on fruit age (days after anthesis, DAA). Control fruit could be utilized up to 80 DAA; samples after that date were judged not to be marketable due to excessive softening. A sample of 48 DAA antisense fruit was enclosed in 4-L glass jars (2 tomatoes per jar) and exposed to a mixture of $100.0 \pm 0.5 \mu \mathrm{L} \cdot \mathrm{L}^{-1}$ ethylene in humidified air utilizing a constant flow-through gas system. The desired ethylene concentration was reached within $1 \mathrm{~h}$ after placing the fruit into the containers and was maintained throughout the imposed ripening period. The flow rate $(1100 \mathrm{~mL} / \mathrm{min})$ selected ensured that $\mathrm{CO}_{2}$ accumulation would not exceed 0.2 $\mathrm{kPa}$; this was checked once a day with an infrared $\mathrm{CO}_{2}$ analyzer (model PIR-2000R; Horiba Instruments, Irvine, Calif.). Defectfree fruit were rinsed with distilled water and dried with paper towels. All chemicals were from Sigma Chemical Co. (St. Louis).

Enzyme extraction and assay. Triplicate composite pericarp samples $(25-100 \mathrm{~g}$, depending on the fruit stage) were homogenized in a Waring blender ( $45 \mathrm{~s}$ ) and then by using a Polytron (45 s) with 3 vol of cold $100 \mathrm{~mm}$ sodium acetate buffer ( $\mathrm{pH} 5$ ) containing $1.4 \mathrm{M}$ $\mathrm{NaCl}, 1 \mathrm{~mm} \mathrm{ZnCl}, 5$ mм 2-mercaptoethanol, and $1.5 \%(\mathrm{w} / \mathrm{v})$ PVPP. The subsequent steps were performed at $4{ }^{\circ} \mathrm{C}$. The suspension was stirred for $30 \mathrm{~min}$, centrifuged at $12000 g_{\mathrm{n}}$ for $15 \mathrm{~min}$, and filtered through glass filter paper (GF/C, Whatman).

Aliquots of filtered extract were assayed for total $a$-Af activity using $p$-nitrophenyl- $\alpha$ D-arabinofuranoside as substrate. Reaction mixtures contained $250 \mu \mathrm{L}$ of $0.1 \mathrm{M}$ citrate buffer, $\mathrm{pH} 4.5,200 \mu \mathrm{L}$ of $0.1 \%$ bovine serum albumin, $50 \mu \mathrm{L}$ of enzyme solution and 200 $\mu \mathrm{L}$ of $13 \mathrm{~mm}$ substrate solution, with incubation at $37{ }^{\circ} \mathrm{C}$. The generation of free $p$ nitrophenol is linear for $3 \mathrm{~h}$ using these reaction conditions. Activities reported are based on rates determined after $1 \mathrm{~h}$ with the reaction stopped by addition of $1 \mathrm{~mL}$ of $0.13 \mathrm{M}$ sodium carbonate. Absorbance was measured at 400 $\mathrm{nm}$. Free $p$-nitrophenol was used as standard.

\section{Results and Discussion}

Preliminary tests showed that the extractability of $\alpha$-Af was salt dependent. The requirement for high $\mathrm{NaCl}$ in the extraction medium suggests that $\alpha$-Af is bound to or at least tightly associated with the cell walls. Tateishi et al. (1996) have reported that extraction of Japanese pear $a$-Af was enhanced using an extraction buffer containing high $\mathrm{LiCl}$, as opposed to $\mathrm{NaCl}$, concentration. However, this approach was not useful for extracting the tomato $\alpha$-Af activity ( $15 \%$ to $20 \%$ of the total activity extracted using $\mathrm{NaCl}$ ). This low recovery of activity is probably due to the instability of the tomato $\alpha$-Af when exposed to dialysis (Sozzi et al., 1998b). Inclusion of the chelators ethylenediamine- tetraacetic acid and trans-1,2-diaminocyclohexane- $N, N$, $N^{\prime}, N^{\prime}$-tetraacetic acid in the extraction buffer did not significantly improve solubilization capacity of the extraction medium.

Previous reports (Campbell et al., 1990; Sozzi et al., 1998b) have indicated that there is little $a$-Af activity in tomato fruit tissues. The protocol used for this experiment strongly enhanced $a$-Af activity recovery, due to inclusion of $\mathrm{Zn}^{2+}$ (activation, stabilization effect, or both), PVPP(removal of phenolic compounds), and 2-mercaptoethanol (maintenance of possible sulfhydryl groups within the active site region in a reduced state) in the extraction medium and the homogenization with a Polytron. The $\mathrm{Zn}^{2+}$ enhancement of $\alpha$-Af activity recovery was generally $10 \%$ to $15 \%$ in pericarp crude extracts.

Our results indicate the presence of $\alpha$-Af early in the development of both control and antisense ACC synthase tomato fruit, with no differences in activity between the two lines (Fig. 1). There are many reports of relatively high activities of a variety of glycosidases early in development of several fruits (e.g., studies with tomato $\alpha$ - and $\beta$-galactosidases, Smith and Gross, 2000; Sozzi et al., 1998a). High activities of $\alpha$-Af and other putative wall-modifying enzymes may reflect functions in wall synthesis and assembly or wall component breakdown to facilitate cell division or growth, or may be unrelated to fruit cell wall metabolism. Early in fruit development, $a$-Af may be modulated by gibberellins or auxins, hormones which tend to be at relatively high concentration at this time (Mapelli et al., 1978). Exogenous IAA and $\mathrm{GA}_{3}$ have been shown to increase $\alpha$-Af activity in intact breaker tomatoes (Sozzi et al., 2000). High $\alpha$ Af activity has also been reported for Japanese pear fruit (Tateishi et al., 1996) during the early stages of development. $\alpha$-Af activity was detected throughout fruit development. Activity declined on a fresh weight basis during the cell expansion phase until maximal fruit size was reached, but total fruit activity increased steadily until 35 DAA and then decreased slightly as the mature green stage was reached (Fig. 1).

During ripening, control fruit expressed steadily increasing levels of $\alpha$-Af activity as the fruit matured to the red ripe stage (Fig. 2) suggesting a role for $\alpha$-Af in the cell wall arabinose loss that parallels fruit softening. In contrast, the $\alpha$-Af activity in antisense fruit decreased after the mature green stage (48 DAA), continuing to fall as the fruit failed to ripen (based on red color development; see Sozzi et al., 1998a). The first color change was seen at the blossom end $32 \mathrm{~d}$ after harvest ( 80 DAA), and $\alpha$-Af activity remained low. Application of $100 \mu \mathrm{L} \cdot \mathrm{L}^{-1}$ ethylene to mature green ACC-S antisense tomatoes caused an increase in $\alpha$-Af activity (Fig. 2). It is well known that initiation and progress of climacteric fruit ripening requires the presence of ethylene and that tomato ripening is associated with cell wall-hydrolase increases (Fischer and Bennett, 1991). Normal ripening fruit and the antisense fruit that have been treated with ethylene show substantial increases in $\alpha$-Af. Therefore, we conclude that the absence of an increase in $\alpha$-Af activity in antisense toma-

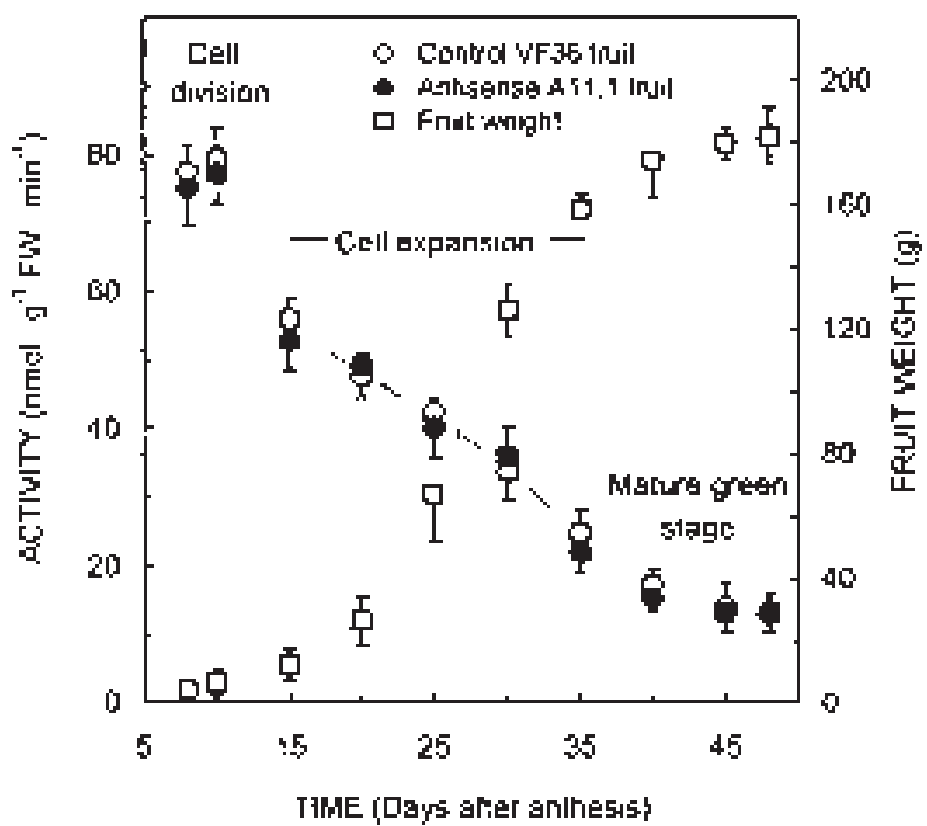

Fig. 1. Total $\alpha$-L-arabinofuranosidase activity and fruit fresh weight during the growth of normal and ACC synthase antisense tomato fruit. Values represent the mean $\pm \mathrm{SD}(\mathrm{n}=3)$. 


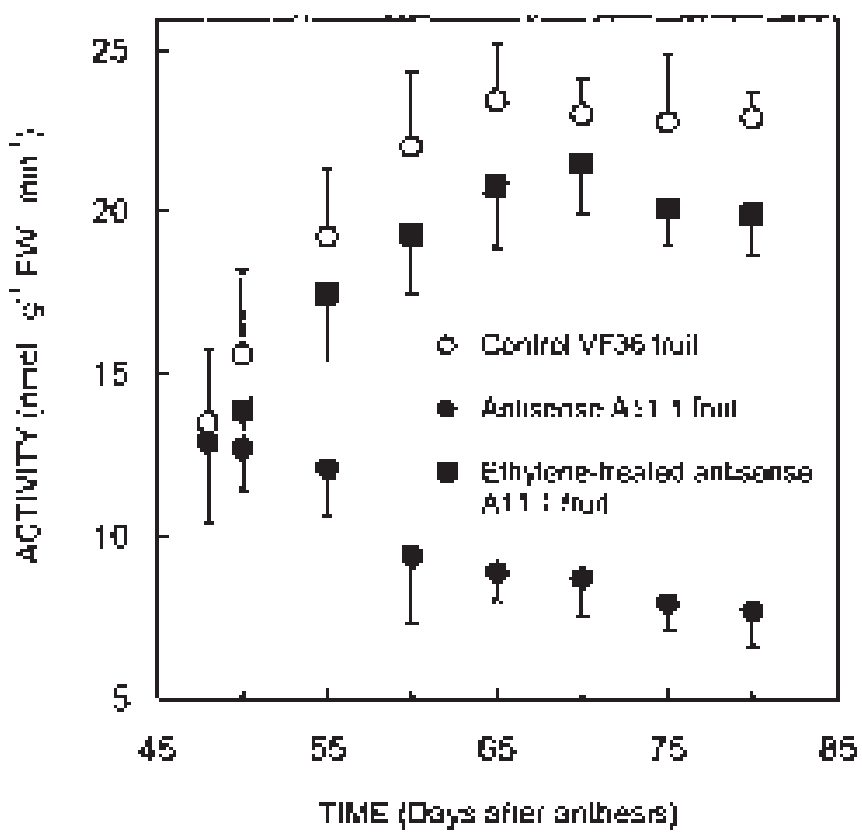

Fig. 2. Total $\alpha$-L-arabinofuranosidase activity during ripening of normal and antisense tomato fruit. Values represent the mean $\pm \mathrm{SD}(\mathrm{n}=3)$. Ripeness stages in normal 'VF36' fruit were established in comparison with the tomato color chart of the USDA (1976), as follows: mature green 4 (day 48); breaker (day 50); light red (day 55); red ripe (day 57); over ripe (day 70). Ethylene treatment of antisense fruit causes them to pass through ripeness stages in the same time frame as normal fruit.

toes, after they reach the mature green stage, reflects the reduction of ethylene synthesis and, subsequently, ethylene action. These results allow us to suggest a role for ethylene in the expression of $\alpha$-Af gene(s), as probably happens with the genes encoding the ripeningrelated enzymes polygalacturonase (Sitrit and Bennett, 1998) and $\beta$-galactosidase II (Smith and Gross, 2000; Smith et al., 1998). Alternatively, ethylene could influence translation of a stable $\alpha$-Af mRNA or activation of an $\alpha$-Af preprotein.

The $\alpha$-Af activity pattern throughout tomato fruit ontogeny is very similar to that of $\beta$-Gal (Sozzi et al., 1998a). For that activity, several isoforms, present at various stages through fruit development, have been identified. However only one ethylene-regulated isoform participates in ripening-related cell wall galactose metabolism(Carey et al., 1995; Carrington and Pressey, 1996; Pressey, 1983; Smith and Gross, 2000; Smith et al., 1998; Sozzi et al., 1998a). This similarity suggests that tomatoes also have more than one $\alpha$-Af isoform and this is the subject of continuing investigation. Of particular interest would be demonstrations that $\alpha$-Af (perhaps a specific isoform) acts on tomato cell wall substrates and that $\alpha$-Af presence correlates with the cell wall arabinose loss that accompanies normal fruit ripening. The antisense ACC synthase fruit could be an excellent system in which to make these tests.

\section{Literature Cited}

Barrett, D.M., E. Garcia, and J.E. Wayne. 1998. Textural modification of processing tomatoes. CRC Crit. Rev. Food Sci. Nutr. 38:173-258.

Brummell, D.A., M.H. Harpster, P.M. Civello, J.M. Palys, A.B. Bennett, and P. Dunsmuir. 1999.
Modification of expansin protein abundance in tomato fruits alters softening and cell wall polymer metabolism during ripening. Plant Cell 11:2203-2216.

Cadahía López, C. 1995. Fertilización, p. 167-187. In: F. Nuez (ed.). El cultivo del tomate. MundiPrensa, Madrid.

Campbell, A.D., M. Huysamer, H.U. Stotz, L.C. Greve, and J.M. Labavitch. 1990. Comparison of ripening processes in intact tomato fruit and excised pericarp discs. Plant Physiol. 94:15821589.

Carey, A.T., K. Holt, S. Picard, R. Wilde, G.A. Tucker, C.R. Bird, W. Schuch, and G.B. Seymour. 1995. Tomato exo-( $1 \rightarrow 4)-\beta$-D-galactanase. Isolation, changes during ripening in normal and mutant tomato fruit, and characterization of a related cDNA clone. Plant Physiol. 108:10991107.

Carpita, N.C. and D.M. Gibeaut. 1993. Structural models of primary cell walls in flowering plants: consistency of molecular structure with the physical properties of the walls during growth. Plant J. 3:1-30.

Carrington, C.M.S. and R. Pressey. 1996. $\beta$-Galactosidase II activity in relation to changes in cell wall galactosyl composition during tomato ripening. J. Amer. Soc. Hort. Sci. 121:132-136.

Cassab, G.I. 1998. Plant cell wall proteins. Annu. Rev. Plant Physiol. Plant Mol. Biol. 49:281-309.

Cheng, G.W. and D.J. Huber. 1997. Carbohydrate solubilization of tomato locule tissue cell walls: Parallels with locule tissue liquefaction during ripening. Physiol. Plant. 101:51-58.

Fischer, R.L. and A.B. Bennett. 1991. Role of cell wall hydrolases in fruit ripening. Annu. Rev. Plant Physiol. Plant Mol. Biol. 42:675-703.

Gross, K.C. and C.E. Sams. 1984. Changes in cell wall neutral sugar composition during fruit ripening: A species survey. Phytochemistry 23:24572461.

Gross, K.C. and S.J. Wallner. 1979. Degradation of cell wall polysaccharides during tomato fruit ripening. Plant Physiol. 63:117-120.
Huysamer, M., L.C. Greve, and J.M. Labavitch. 1997. Cell wall metabolism in ripening fruit. IX. Synthesis of pectic and hemicellulosic cell wall polymers in the outer pericarp of mature green tomatoes (cv XMT-22). Plant Physiol. 114:15231531.

Konno, H., Y. Yamasaki, and K. Katoh. 1987. Purification of an $\alpha$-L-arabinofuranosidase from carrot cell cultures and its involvement in arabinoserich polymer degradation. Physiol. Plant. 69:405412.

Mapelli, S., C. Frova, G. Torti, and G.P. Soressi. 1978. Relationship between set, development and activities of growth regulators in tomato fruits. Plant Cell Physiol. 19:1281-1288.

Pressey, R. 1983. $\beta$-Galactosidases in ripening tomatoes. Plant Physiol. 71:132-135.

Priem, B., R. Gitti, C.A. Bush, and K.C. Gross. 1993. Structure of ten free $\mathrm{N}$-glycans in ripening tomato fruit. Arabinose is a constituent of a plant $N$ glycan. Plant Physiol. 102:445-458.

Redgwell, R.J., M. Fischer, E. Kendal, and E.A. MacRae. 1997. Galactose loss and fruit ripening: High-molecular-weight arabinogalactans in the pectic polysaccharides of fruit cell walls. Planta 203:174-181.

Saha,B.C. 2000. $\alpha$-L-Arabinofuranosidases: biochemistry, molecular biology and application in biotechnology. Biotechnol. Adv. 18:403-423.

Seymour, G.B., I.J. Colquhoun, M.S. DuPont, K.R Parsley, and R.R. Selvendran. 1990. Composition and structural features of cell wall polysaccharides from tomato fruits. Phytochemistry 29:725-731.

Sitrit Y. and A.B. Bennett. 1998. Regulation of tomato fruit polygalacturonase mRNA accumulation by ethylene: A re-examination. Plant Physiol. 116:1145-1150.

Smith, D.L. and K.C. Gross. 2000. A family of at least seven $\beta$-galactosidase genes is expressed during tomato fruit development. Plant Physiol. 123:1173-1183.

Smith, D.L., D.A. Starrett, and K.C. Gross. 1998. A gene coding for tomato fruit $\beta$-galactosidase II is expressed during fruit ripening. Cloning, characterization, and expression pattern. Plant Physiol. 117:417-423.

Sozzi, G.O., S.A. Camperi, O. Cascone, and A.A. Fraschina. 1998a. Galactosidases in tomato fruit ontogeny: Decreased galactosidase activities in antisense ACC synthase fruit during ripening and reversal with exogenous ethylene. Aust. J. Plant Physiol. 25:237-244.

Sozzi, G.O., S.A. Camperi, A.A. Fraschina, and O. Cascone. 1998b. Assessment of different treatments for the concentration of three tomato fruit glycosidases from crude extracts. Biotechnol. Tech. 12:645-647.

Sozzi, G.O., G.D. Trinchero, and A.A. Fraschina. 2000. Ethylene and glycosidase promotion in breaker $\mathrm{GA}_{3}-$ and IAA-treated tomato fruit (Lycopersiconesculentum Mill.). J. Plant Growth Regul. 19:359-368.

Tateishi, A., Y. Kanayama, and S. Yamaki. 1996. $\alpha$ L-Arabinofuranosidase from cell walls of Japanese pear fruits. Phytochemistry 42:295-299.

Trinchero, G.D., G.O. Sozzi, A.M. Cerri, F. Vilella, and A.A. Fraschina. 1999. Ripening-related changes in ethylene production, respiration rate and cell-wall enzyme activity in goldenberry (Physalis peruviana L.), a solanaceous species. Postharvest Biol. Technol. 16:139-145.

USDA. 1976. United State standards for grades of fresh tomatoes. U.S. Dept. Agr., Agr. Mktg. Serv., Washington, D.C., $10 \mathrm{p}$.

York, W.S., V.S.K. Kolli, R. Orlando, P. Albersheim, and A. Darvill. 1996. The structures of arabinoxyloglucans produced by solanaceous plants. Carbohyd. Res. 285:99-128. 